\title{
An Interactive Approach for QualNet-based Network Model Evaluation and Testing at Real Time
}

\author{
Peng Gong*, Mingguan Li*, Jiejun Kong**, Ping Li*, and Duk Kyung Kim*** \\ * National Key Laboratory of Mechatronic Engineering and Control, School of Mechatronical Engineering, \\ Beijing Institute of Technology, Beijing, China \\ ** Department of Computer Sciences, University of Florida, Gainesville, FL, USA \\ *** Department of Information and Communication Engineering, Inha University, Incheon, Korea \\ penggong@bit.edu.cn, liming.Img@gmai.com,jkong@cs.ucla.edu, liping85@bit.edu.cn, kdk@inha.ac.kr
}

\begin{abstract}
Network simulators, e.g., QualNet, have been widely used for the sake of cost efficiency in network model evaluation and testing. In order to evaluate and test the impacts of parameters on network models, massive simulations and result analysis are typically running repetitively in a sealed virtual environment per a non-real-time simulated clock, apart from real-time human interactions. The major drawback of this caustic approach is obvious in large-scale network simulations with long simulation time and hardware-in-the-loop accessories, when the result analysis must be adaptive to human factors in nowadays computer networks. In this paper, we design and implement an interactive real-time user interface (RTUI), which is a reliable human-in-the-loop framework that allows human users to dynamically modify the parameters of network model during the simulation process. With negligible delays and overhead, the impacts of parameter changing on network model can be actualized and measured at real time, rather than in a repetitive manner of the conventional approach, resulting in RTUI's time-efficiency and cost-efficiency in network protocol debugging and assessment.
\end{abstract}

Keywords - RTUI; Network model evaluation\&testing; QualNet

\section{INTRODUCTION}

Network model evaluation and testing are essential in improving various new wireless and heterogeneous communication technologies [1-4] and their deployment in the real world [5-8]. Evaluation and test of a network model generally can be classified into two major types: physical test approach and simulation test approach [9]. In the case of physical test, the network model runs on many test-beds and their performance is evaluated over resource demanding experiment rounds, which is costly and very limited for specific test scenarios [10]. Compared to the physical test, the simulation test approach is more cost-effective and flexible, since none of the actual test-beds is required and the experimentations can be conducted in more scalable network scenarios. In particular, the simulation test approach is the only viable approach for large-scale experiments or simulations due to prohibitively high expense incurred by actual physical tests. However, the simulation test approach still needs to catch up with the physical test approach in regards to many real-time demands including the interactive human factors. Although a back-end support tool[11] for large-scale parameter configuration has been proposed to evaluate the network performance for a particular setting of protocol parameters, the proposed solution does not apply to large-scale real-time simulations, for example, in a network comprised of thousands of ad hoc routers simulated by QualNet. A NS-2 based game environment has been designed to train people in terms of network management skills, however, the proposed solution does not apply to wireless networks since NS platforms lack the wireless signal processing models presented in QualNet [12] and OPNET [13].

In this paper, RTUI is proposed for the QualNet-based simulator to serve large-scale wireless network model evaluation and testing. A reliable human-in-the-loop framework is achieved between graphical user interface (GUI) and QualNet-based simulator so that the parameters of network model in simulator can be dynamically and manually changed by human users. With negligible delays and overhead, the impact of parameters on the network model is actualized at real time during simulation, rather than by repetitions. Experiment results demonstrate the efficiency of the proposed RTUI.

The rest of the paper is organized as follows. Section II describes the real-time user interface in detail. In Section III, the proposed RTUI approach is evaluated by intensive experiments. Finally, the conclusions are drawn in Section IV.

\section{Proposed Real-Time USER Interface}

\section{A. Real-Time User Interface design}

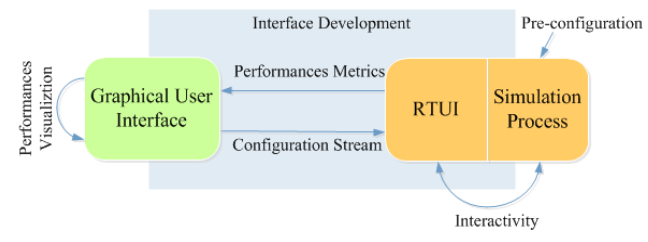

Figure 1. Structure of RTUI framework

The proposed RTUI framework is composed of RTUI GUI, and QualNet-based simulator as shown in Fig. 1. The RTUI is 
investigated as an external interface of QualNet and allows the QualNet to interact with external entities such as the program on local host or remote host, e.g., a GUI. The development of RTUI follows the QualNet modelling framework with initialization, event dispatcher, and finalization. The initialization is responsible for interface parameters configuration and UDP socket communication between GUI and QualNet-based simulator. The event dispatcher emulates the behaviour and state transition of network models in the simulator. The function of finalization is utilized to release the memory, close the socket, and print the statistics of simulation.

The main functions of RTUI are to receive the incoming configuration stream from GUI, modify the relative parameters in the network model of simulator and forward the performance metrics to GUI for visualization. In the framework, a connection is established via UDP sockets communication between GUI and simulator. When human user makes a change on particular parameters of network model on the GUI, a corresponding configuration stream is sent to RTUI and then, the specified parameter of network model is changed in the simulator. In addition, in order to analyse the impact of the parameter change, the performance metrics will be periodically fed back to the GUI.

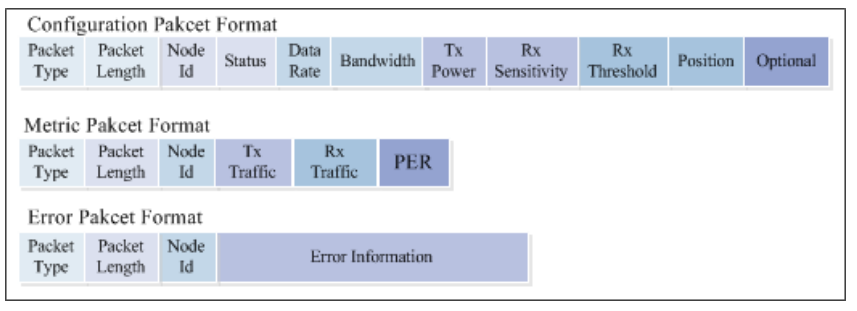

Figure 2. Packet formats to communicate the model

Fig. 2 shows the defined packet formats in the RTUI. If any parameters of network model have been changed on the GUI, a configuration packet or stream is sent to the RTUI. This message contains a series of segments, e.g., the packet type, the packet length, the node identification, the interface status, the data rate, the other transmission or reception parameters, and the node coordinates. Regular network performances, e.g., statistic of received or transmitted packets, Packet Error Rate (PER), and delay, are fed back to GUI as the metric packets. A metric packet contains the packet type, the packet length, and the node identification. RTUI also can indicate the error information, when the human user makes any incorrect operation in the GUI.

Figure 3 shows the working procedure flowchart of RTUI and the detailed description is given as following:

- RTUI listens to a UDP port to receive the incoming configuration stream from the GUI, which is stored in the receive buffer.

- Then the packet parser is informed to parse the packet at the top of receive buffer. The processing order of receive buffer is first in first out (FIFO), ensuring that the required timing constraints are satisfied.

- As soon as the parsing is finished, the node manager is scheduled. The node manager is responsible for the management of the nodes in the simulation scenario.
- If the node specified by the packet exists in the simulation scenario, event dispatcher is called. Otherwise the packet is dropped immediately.

- According to the node identification indicated in the packet, the event dispatcher assigned the event processor of the specified node to handle this packet.

- The event processor extracts the parameter segments from the packet and modifies the specified parameters of the network model or the mobility model. The other relevant variables of the network model have to be recalculated based on these changing.

- The event processor schedules a timer event periodically. When the timer event is scheduled, the performances recorded in simulation process are encapsulated as a packet and put in the send buffer.

- Then the send buffer pushes it to the GUI via socket communication. The packet parse, node manager, and event dispatch are only involved in the processing of incoming traffic to the simulator.

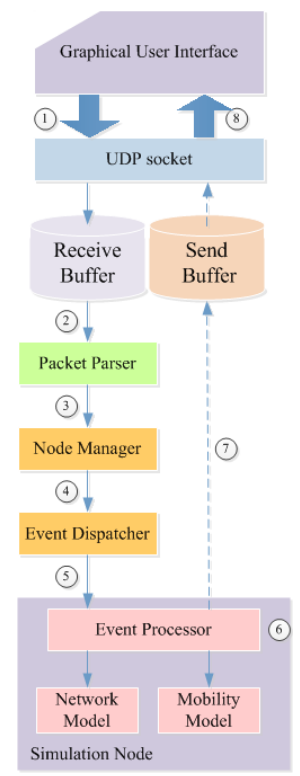

Figure 3. Working procedure of RTUI

\section{B. Simple Graphical User Interface}

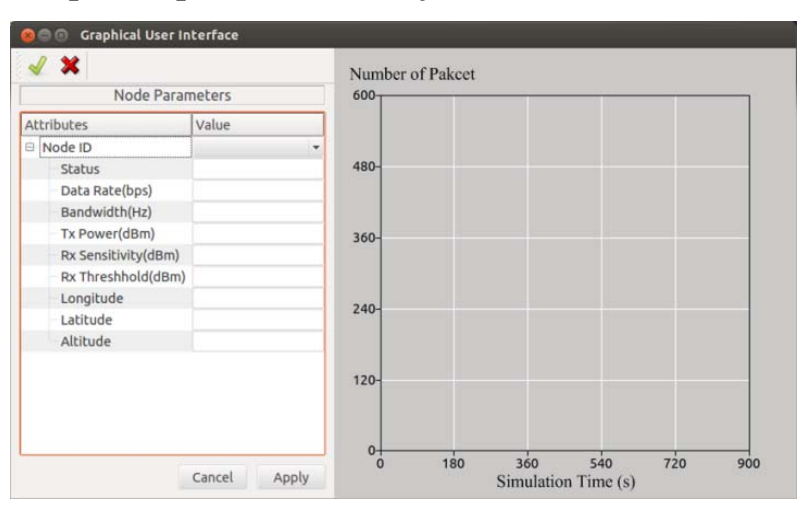

Figure 4. Simple Graphical User Interface for experiments 
Figure 4 shows a simple graphical user interface, which here is only developed to provide convenient interactivity between human user and simulator as well as the visualization of the packet-level performances at real time. On the left side of GUI, the parameters of node are displayed. In this simple GUI, only a few parameters of physical layer model are involved, but the proposed RTUI framework can also support the other network layer, e.g., transport layer, network layer, and MAC layer. On the right side of GUI, the graph shows the curves of performances with different colors. When the connection is established between GUI and QualNet, the RTUI sends the performances metric of network model to GUI and GUI displays them to the human user. When the parameters of network model are mutually changed by the operator, GUI can easily keep track of network performances and show how the parameter change affects the performances of network model.

\section{III.EXPERIMENTS AND RESULTS}

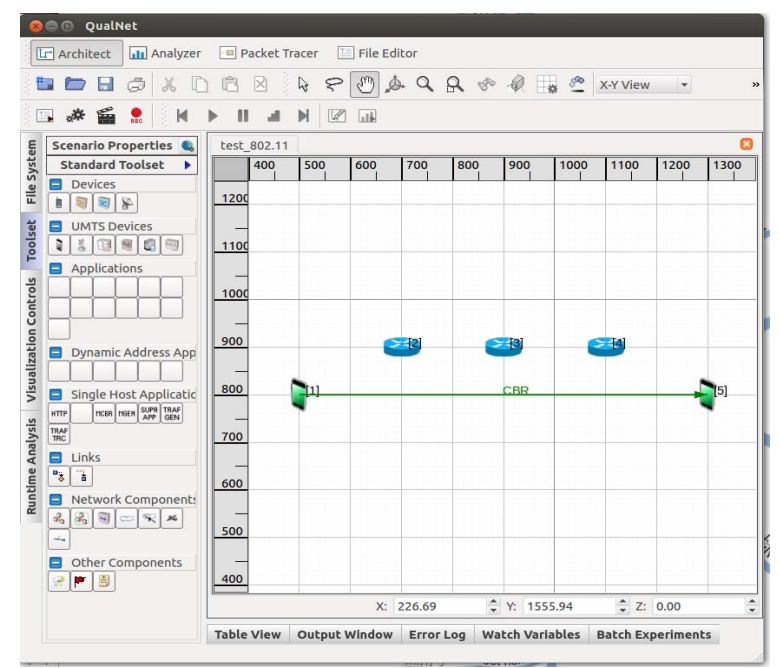

Figure 5. Pre-configured network model in QualNet-based simulator

Figure 5 shows an ad-hoc network model in a QualNet based simulator. For simplicity, a simple data transmission model from node 1 to node 5 is pre-configured, while nodes 2 , 3 , and 4 are considered as routers and the routing path for its data transmission is $1 \rightarrow 2 \rightarrow 3 \rightarrow 4 \rightarrow 5$. The transmit power and received threshold of all the nodes are pre-defined as $15 \mathrm{dBm}$ and $-81 \mathrm{dBm}$, respectively. In order to show the efficiency of proposed RTUI framework, we first test the RTT between GUI and QualNet to check the delay to the simulator, and then, we change the transmit power, received threshold, and position of node by the GUI through the RTUI.

Figure 6 shows the round trip delay time (RTT) between GUI and QualNet-based simulator. Due to the additional command stream, processing time, and performances visualization, RTUI may introduce some delays and overhead to the simulator. However, experiment result demonstrates that the averaged RTT introduced by the RTUI is just $66.5 \mathrm{us}$ [14] and is actually negligible for the QualNet-based simulator, which implies that the RTUI almost does not change the fidelity of network simulator.

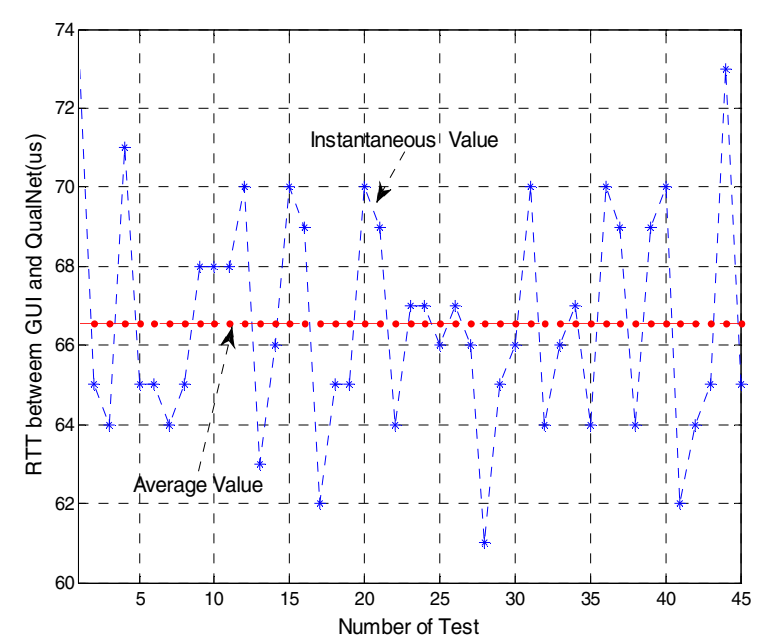

Figure 6. Round trip delay time of RTUI between GUI and QualNet

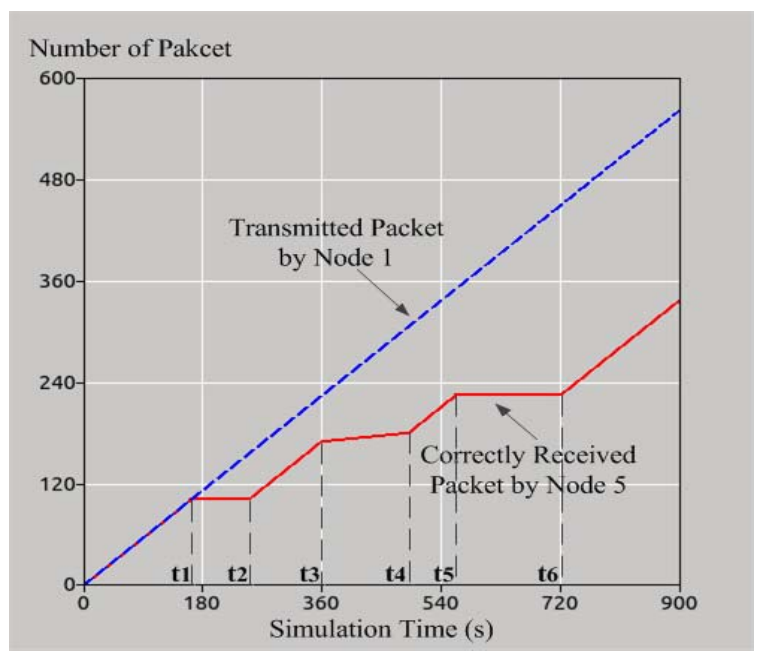

Figure 7. Performances of correctly received packets at node 5 with different transmit power, received threshold, or position at node 4 .

TABLE 1. ACTION AT GUI FOR PARAMETER CHANGING OF NODE 4 AT DIFFERENT SIMULATION TIME

\begin{tabular}{|c|l|}
\hline Time & \multicolumn{1}{|c|}{ Action at GUI for parameter changing } \\
\hline 0 & Simulation begins \\
\hline t1 & Transmit power is changed from $15 \mathrm{dBm}$ to $1 \mathrm{dBm}$ \\
\hline t2 & Transmit power is changed from $1 \mathrm{dBm}$ to $15 \mathrm{dBm}$ \\
\hline t3 & Reception threshold is changed from $-81 \mathrm{dBm}$ to $-50 \mathrm{dBm}$ \\
\hline t4 & Reception threshold is changed from $-50 \mathrm{dBm}$ to $-81 \mathrm{dBm}$ \\
\hline t5 & Y-coordinate is changed from 900 to 600 \\
\hline t6 & Y-coordinate is changed from 600 to 900 \\
\hline $\mathbf{9 0 0}$ & Simulation ends \\
\hline
\end{tabular}

Figure 7 shows performances of correctly received packets at node 5 with different transmit power, received threshold, or position at node 4 , which is achieved by manually modify the corresponding parameters of network model by the GUI via the RTUI. The detail procedure of human action at GUI is listed in Table 1. At time zero, the Node 1 transmits the packet to the node 5 at a constant data rate, thus, the gradient of its transmitted packets is constant from 0 to $t 1$. At $t 1$, the transmit 
power of node 4 is manually reduced from $15 \mathrm{dBm}$ to $1 \mathrm{dBm}$. Due to a lower transmit power at node 4 , the link from node 4 to node 5 is broken and the gradient of correctly packet becomes zero. However, when the transmit power is changed back to $15 \mathrm{dBm}$, nodes 4 and 5 are connected at $\mathrm{t} 2$ and the corresponding gradient of correctly received packets at node 5 become larger, which is similar as that at beginning. At $\mathrm{t} 3$, the reception threshold of node 4 is re-set to $-50 \mathrm{dBm}$, which results in a poor link quality from node 3 to node 4 . Thus, the number of correctly received packets at node 5 becomes smaller, and the corresponding gradient becomes smaller. When the reception threshold of node 4 is changed to $-81 \mathrm{dBm}$, the data transmission returns to normal. In addition, we also changed the position of node 4 as shown in Table 1 and Fig. 7 , similar result can be observed. Therefore, the experiment results have demonstrated that with proposed RTUI the parameter of network model, e.g., the transmit power, reception threshold, and position of node, in QualNet-based simulator can be dynamically changed and the corresponding impact can be obviously realized at real time.

\section{IV.CONCLUSIONS}

In this paper, a new real time user interface is investigated for the QualNet-based network evaluation and testing. With the aid of RTUI, a reliable human-in-the-loop framework is accomplished so that the human factors such as the GUI programs on local host or remote host can interact with QualNet-based simulator and changes the pre-configured parameter or condition of the network model to realize the impact of such parameter in the measurements. Experiment results demonstrate that the investigated RTUI works efficiently and the parameter or condition of the network model can be manually and dynamically changed, leading to better network model evaluation and function debugging.

\section{ACKNOWLEDGMENT}

This research was supported in part by National Natural Science foundation of China (No.61201180), Beijing Natural Science Foundation (N0.4132055), Excellent Young Scholars Research Fund of Beijing Institute of Technology, and Basic Science Research Program through the NRF (NRF2013R1A1A2A10059215). Corresponding author: Peng Gong.

\section{REFERENCES}

[1] J. W. Wu, C. W. Cheng, S. R. Yang, C. H. Gan, Y. B. Chen, "uLIPA A universal local IP access solution for 3GPP mobile networks," in Proc. IEEE Wireless Communications and Mobile Computing Conference, pp. 1235-1240, Italy, July, 2013.

[2] K. P. Makhecha, K. H. Wandra, " $4 \mathrm{G}$ Wireless Networks: Opportunities and Challenges," in Proc. IEEE INDICON, pp. 1-4, India, Dec, 2009.

[3] V. A. Siris, E. Z. Tragos, N. E. Petroulakis, "Experiences with a metropolitan multiradio wireless mesh network: design, performance, and application," IEEE Communications Magazine, vol. 50, no.7, pp. 128-136, July, 2012.

[4] K. Kashiki, T. Suzuki, and A. Yamaguchi, "Testbed development and performance improvement of heterogeneous radio networks," IEEE Communications Magazine, vol. 50, no. 9, pages. 186-192, Sep., 2012.

[5] S. Begum, A. Helmy, and S. Gupta, "Modeling and test generation fo worst-case performance evaluation of MAC protocols for wireless ad hoc networks," in Proc. IEEE International Symposium on Modeling,
Analysis \& Simulation of Computer and Telecommunication Systems, pp. 1-10, U.K., Sep., 2009.

[6] Y. Chen, L. Hou, Y. Liu, Y. Zhang, and F. Qi, "Study and implementation of model-driven testing method for network management interface," in Proc. IEEE International Conference on Communications Technology and Applications, pp. 259-263, China, Oct., 2009.

[7] Y. Chen, Z. Wang, Y. Liu, F. Qi, and L. Meng, "Network management interface automatic testing framework based on model-driven," in Proc International Conference on Advanced Intelligence and Awareness Internet, pp. 72-76, China, Oct., 2010,

[8] C. Xing, G. Zhang, and M. Chen, "Research on universal network performance testing model," in Proc. IEEE International Symposium on Communications and Information Technologies, pp. 780-784, Australia, Oct., 2007.

[9] T. Z. Li, J. Kong, P. Li, and P. Gong, "RVIP: bridging live networks and software virtual networks for large scale network simulation at real time," in Proc. IEEE $15^{\text {th }}$ ACM International Conference on Modeling, Analysis and Simulation of Wireless and Mobile Systems, pp. 171-178, Cyprus, Oct., 2012

[10] A. Alvarez, R. Orea, S. Cabrero, and et al., "Limitations of network emulation with single-machine and distributed ns-3," in Proc. IEEE $3^{\text {rd }}$ International Conference on Simulation Tools and Techniques, no. 67, Spain, Mar., 2010.

[11] T. Ye, H. T. Kaur, S. Kalyanaraman, and M. Yuksel, "Large-scale network parameter configuration using an on-line simulation framework," IEEE ACM Transactions on Networking, vol. 16, no. 4, pp. 777-790, August 2008.

[12] E. Arslan, M. Yuksel, M. H. Gunes, "Network management game," in Proc. IEEE 18th Workshop on Local \& Metropolitan Area Networks, pp. 1-6, U.S.A, Oct., 2011.

[13] B. Allan, A. Jasmine, R. Josiane, C. Allan, F. Ramz, G. Hermínio, F Simone, C. Bruno, C. Gervásio, "Implementation of a new propagation model for 5.8GHz systems in OPNET Simulator," in Proc. IEEE 7th European Conference on Antennas and Propagation, pp. 935-938, Sweden, April 2013

[14] T. Staub, R. Gantenbein, and T. Braun, "Virtualmesh: an emulation framework for wireless mesh networks in OMNet++," Simulation, vol. 87, no. 1-2, pp. 66-81, Jan., 2011.

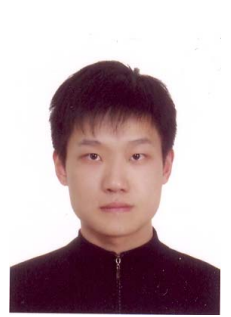

Peng Gong received the BS degree in Mechatronical Engineering from Beijing Institute of Technology, Beijing, China, in 2004, and the MS and Ph.D. degrees from the Inha University, Korea, in 2006 and 2010, respectively. In July 2010, he joined the School of Mechatronical Engineering, Beijing Institute of Technology, China. His research interests include link/system level performance evaluation and radio resource management in wireless systems, information security, and the next generation wireless systems such as 3GPP LTE, UWB, MIMO, Cognitive radio and so on.

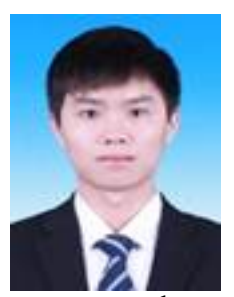

Mingguan $\mathrm{Li}$ received the $\mathrm{BS}$ degree in Mechatronical Engineering from Beijing Institute of Technology in 2011, and now he is a MS candidate in School of Mechantronical Engineering, Beijing Institute of Technology. $\mathrm{He}$ is going to join the Samsung Guangzhou Mobile R\&D Center (SGMC) in china. His research interests include wireless network simulation and emulation, and development of efficient, scalable, and secure network protocols for wireless networks.

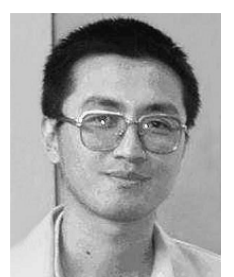

Jiejun Kong received the Ph.D. degree in computer science from the University of California, Los Angeles, in 2004. He was a senior researcher in Scalable Network Technologies, Inc. and a post-doctoral researcher in the Network Research Lab of Computer Science Department at UCLA. He is interested in developing efficient, scalable, and secure network protocols for wireless networks. His research topics include secure and anonymous routing, authentication, access control, 
distributed data harvesting, and network security modeling in mobile wireless networks, in particular, those with challenging network constraints and high security demands, such as mobile ad hoc networks and underwater sensor networks. He has contributed to the design, implementation, and testing of network protocols within the NSF iMASH, ONR MINUTEMAN/STTR, NSF WHYNET and all QualNet/EXata commercial software development projects. He is now collaborating with Beijing Institute of Technology and Southeast University on wireless networking research.

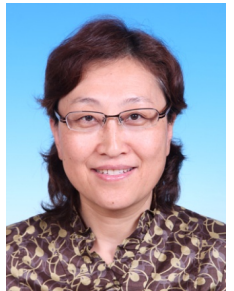

Ping Li received the B.S. and M.S. degree in Mechant ronical Engineering from Dalian Jiaotong University, Dalian, China, in 1985 and 1987, respectively, and the Ph.D. degrees from the Beijing Institute of Technolo gy, China, in 1995. In Sept. 1996, she joined School o f Mechatronical Engineering, Beijing Institute of Tec hnology, China. Her research interests include inform ation countermeasure in wireless systems, information security, terminal information resistance and information security in wireless communication.

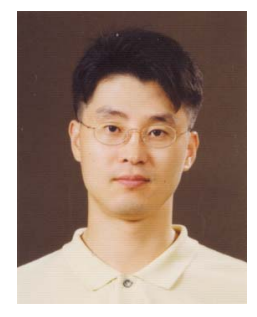

Duk Kyung Kim received the B.S. degree in Electrical Engineering from Yonsei University, Seoul, Korea, in 1992, and the M.S. and Ph.D. degrees from the Korea Advanced Institute of Science and Technology (KAIST), in 1994 and 1999, respectively. From 1999 to 2000, he was a postdoctoral researcher at the Wireless Laboratories, NTT DoCoMo, Japan. From 2000 to 2002, he worked at R\&D center, SK Telecom, Korea and involved in the standardization in $3 \mathrm{GPP}$ and also in $4 \mathrm{G}$ system development. In March 2002, he joined Inha University, Korea. His research interests include system performance evaluation at link/system level, radio resource management, and multi-media provision in wireless systems and next generation wireless systems such as 3GPP LTE, Wibro, UWB, MIMO, Cognitive radio and so on. 